in vivo $34: 3597-3600(2020)$

doi:10.21873/invivo.12204

\title{
Bilateral Primary Marginal Zone Lymphoma of the Breast - A Rare Breast Malignancy
}

\author{
SWAPNA TALLURI ${ }^{1}$, CHARUMATHI RAGHU SUBRAMANIAN ${ }^{2}$, \\ ELIZABETH WERNER ${ }^{3}$, VINEELA KASIREDDY ${ }^{4}$ and LOWRY PHILIP ${ }^{4}$ \\ ${ }^{1}$ Department of Medicine, Guthrie Clinic/Robert Packer Hospital, Sayre, PA, U.S.A.; \\ ${ }^{2}$ Washington Hospital Healthcare System, Fremont, CA, U.S.A.; \\ ${ }^{3}$ Department of Radiology, Guthrie Clinic/Robert Packer Hospital, Sayre, PA, U.S.A.; \\ ${ }^{4}$ Department of Hematology and Oncology, Guthrie Clinic/Robert Packer Hospital, Sayre, PA, U.S.A.
}

\begin{abstract}
Extranodal marginal zone lymphoma (EMZL) presents only rarely within the breast, although the incidence of breast EMZL has increased in the past decade for unclear reasons. Due to its rarity, the etiology, course, and treatment response of this cancer are less studied. Case Report: We present the case of a 64-year-old female who had bilateral diffuse irregularity in a trabecular pattern on screening mammogram. Random ultrasound-guided breast biopsy of the right breast demonstrated an extra-nodal marginal zone $B$ cell lymphoma. She also had approximately $25 \%$ marrow involvement by mucosa-associated lymphoid tissue-type marginal zone lymphoma and splenomegaly. Clinically she remained asymptomatic during a 1-year follow-up. Although she presented with advanced-stage disease involving both breasts, spleen and bone marrow, given her lack of associated symptoms, she was observed with active surveillance. Conclusion: Asymptomatic cases of breast EMZL can be managed with close observation as exemplified by our case.
\end{abstract}

Extranodal marginal zone lymphoma (EMZL), also known as mucosa-associated lymphoid tissue (MALT) lymphoma, arise only rarely within the breast, although the incidence of breast EMZL has increased in the past decade for unclear reasons. Due to its rarity, the etiology, course, and treatment response of this cancer are less well-studied. Here we present a case of bilateral primary breast EMZL. Patient consent was acquired for reporting this case.

This article is freely accessible online.

Correspondence to: Swapna Talluri, MD, 1 Guthrie Square, Department of Medicine, Guthrie Clinic/Robert Packer hospital, Sayre, PA 18840, U.S.A. E-mail: Swapnatalluri3@gmail.com

Key Words: Breast lymphoma, breast MALT, extranodal lymphoma, breast cancer, asymptomatic lymphoma.

\section{Case Report}

A 64-year-old female with no significant past medical or family history underwent screening mammogram, which showed developing bilateral diffuse fine nodularity in trabecular pattern and was new compared to prior mammograms as shown in Figure 1. She did not have palpable breast masses, skin thickening or axillary adenopathy. Bilateral ultrasound of the breasts showed no discrete mass, distortion, adenopathy or skin thickening. However, there were numerous echogenic lesions of $1 \mathrm{~mm}$ scattered throughout the breast, best seen in the fat. Magnetic resonance imaging of the breast showed no abnormal enhancing mass or adenopathy. Random ultrasound-guided breast biopsy of the right breast demonstrated an extra-nodal marginal zone B-cell lymphoma. Core biopsy of fibroadipose tissue showed patchy lymphoid aggregates consisting of small atypical lymphocytes with pale cytoplasm as shown in Figure 2. The atypical lymphocytes were positive for CD20 and BCL2 apoptosis regulator (BCL2) and negative for CD3, CD5, CD10, CD23, BCL6 transcription repressor (BCL6), and cyclin D1 as shown in Figure 3. Bone marrow biopsy from right iliac crest showed approximately $25 \%$ marrow involvement by mucosa-associated lymphoid tissue (MALT)type marginal zone lymphoma. The lymphocytes were positive for CD 20, CD43, and BCL2 and negative for CD3, CD5, CD10, CD23, BCL6, and cyclin D1.

The patient had a palpable cervical lymph node, and two nodules each around $3 \times 4 \mathrm{~mm}$ at the medial end of both upper and lower right eyelids adjacent to the lacrimal caruncle on the physical examination which were diagnosed later as sebaceous cysts. Magnetic resonance imaging of the orbits showed no significant findings. computed tomography of the chest, abdomen and pelvis showed splenomegaly around 15 $\mathrm{cm}$ and no significant adenopathy. Follow-up mammogram 1 year later showed continued interval increase in the bilateral diffuse fine nodularity in a trabecular pattern in both breasts. 

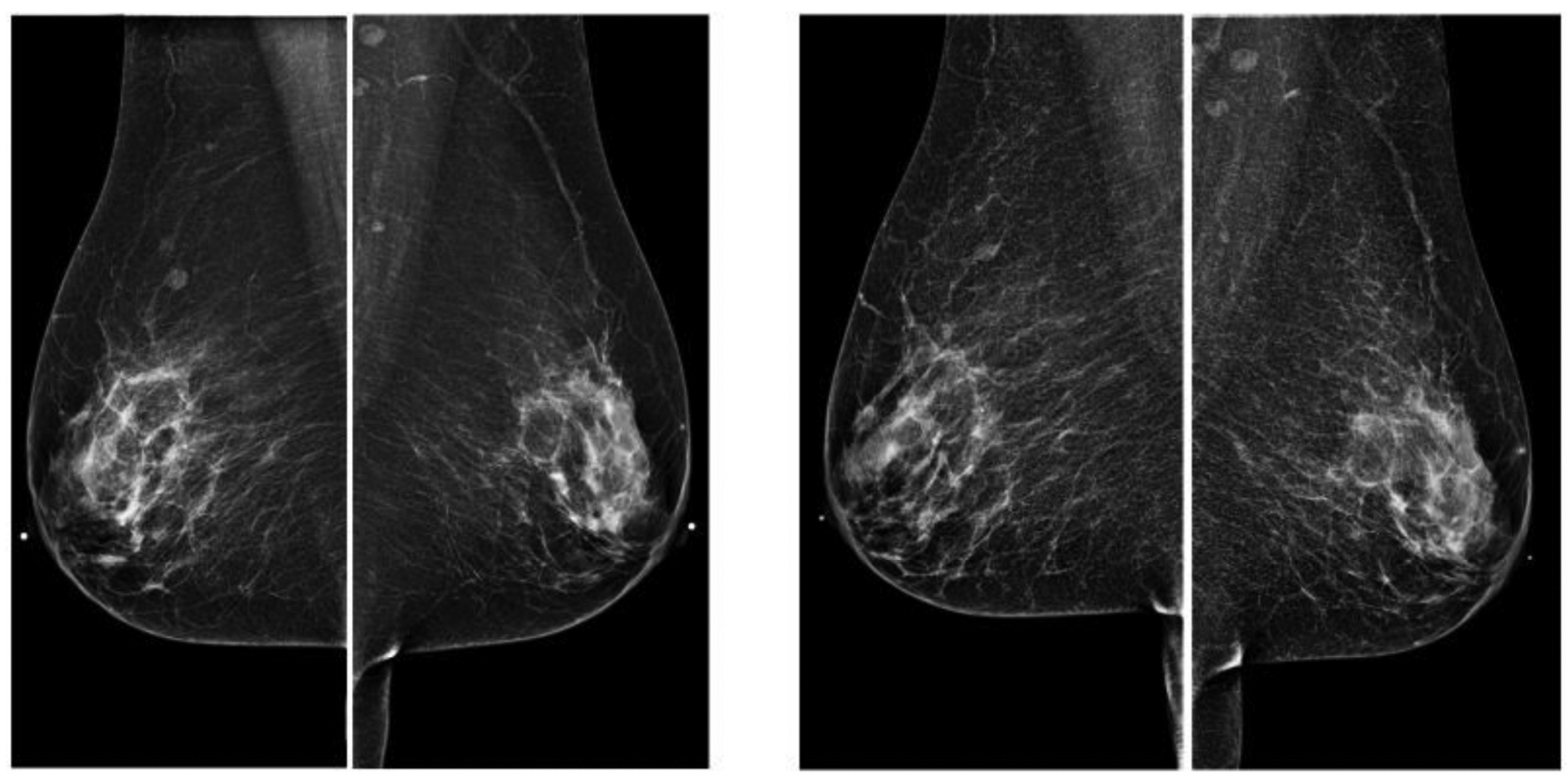

Figure 1. Screening mammogram showing bilateral diffuse irregularity (right) new compared to a prior mammogram (left).

Clinically, she remained asymptomatic during the 1-year follow-up duration. Her MALT international prognostic index was intermediate. Although she presented with advancedstage disease involving both breasts, spleen and bone marrow, given her lack of associated symptoms she was observed with active surveillance. We will consider low-dose irradiation if this patent develops any local symptoms, or rituximab for systemic problems.

\section{Discussion}

Primary breast lymphoma, which comprises less than $1 \%$ of all non-Hodgkin's lymphomas, is a rare type of breast malignancy. The most common histological subtype of primary breast lymphoma is diffuse large B-cell lymphoma. The second most common is low-grade marginal zone B-cell lymphoma (MZL) (1), accounting for $9 \%$ of primary breast lymphoma cases (2). Marginal zone lymphoma has three subtypes: Splenic, extranodal, and nodal. EMZL arise from mucosal sites, with the most frequent being the stomach, lung, thyroid, skin, and salivary and lacrimal glands (2). Primary breast EMZL has been increasing in incidence in the past decade for unclear reasons, even in women younger than 50 years of age (3). To date, there have been no prospective studies on this rare type of cancer.

The etiology of primary breast EMZL is unclear. Exogenous hormone therapy has not shown any strong association with primary breast EMZL (4). Anaplastic largecell histological type of primary breast lymphoma is reported more frequently in individuals with breast implants, but no such association is significant with EMZL (1). Several infectious agents are known to act as inflammatory triggers for EMZL at other sites, such as Helicobacter pylori in gastric MZL (3). An association between autoimmune diseases and EMZL at other sites has also been established such as Hashimoto's thyroiditis and Sjogren's syndrome in thyroid and salivary EMZL, respectively (5). No such infectious agents, autoimmune diseases, or genetic abnormalities have been consistently associated with primary breast EMZL.

Most cases of breast lymphoma present as a palpable breast mass and some are detected incidentally on screening mammography (1), with a median age at diagnosis of 61-65 years (range $=23-88$ years) $(1,2)$. Around $85 \%$ of breast EMZL was diagnosed in postmenopausal women (2). Bilateral involvement was seen in a few cases (1).

The initial steps in diagnosis are usually diagnostic mammography or ultrasound followed by core needle biopsy or surgical biopsy to establish a histological diagnosis (2). Breast lymphoma frequently appears as a noncalcified oval or round mass which either has circumscribed or indistinct margins on mammogram $(6,7)$.

Primary breast EMZL is less aggressive than other forms of breast lymphoma and overall has good outcomes. It shows a good response to initial radiotherapy, and the majority of cases do not require extensive surgery $(2,4)$. Ludmir et al. reported a good response rate with ultra-low dose radiotherapy of $4 \mathrm{~Gy}$ with results comparable to the standard dose of $30 \mathrm{~Gy}$. Breast EMZL also responds very well to single-agent rituximab therapy (4). In the series of Ludmir 

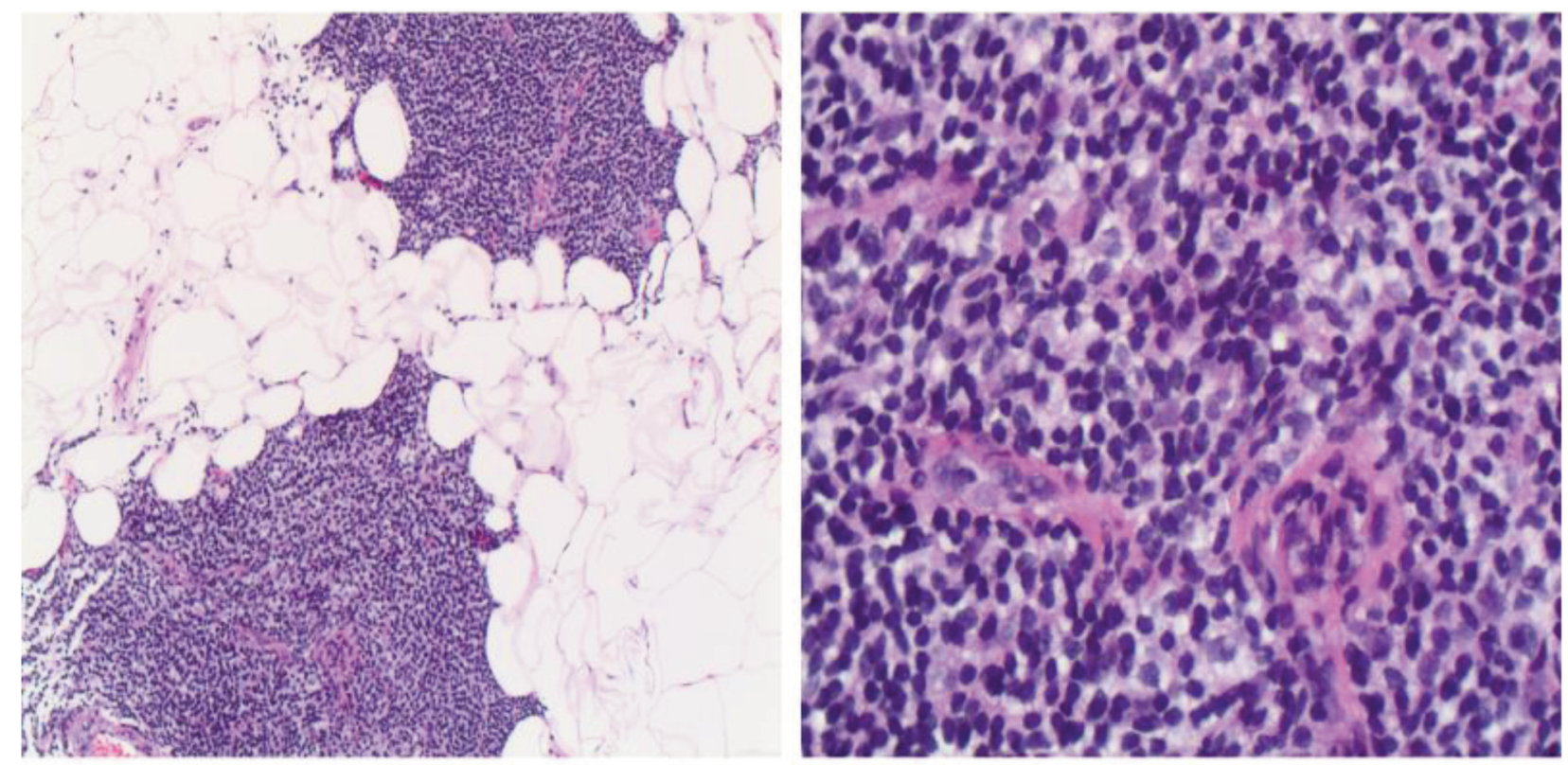

Figure 2. Core biopsy of fibro-adipose tissue showing nodular collection of small lymphoid aggregates on the left [hematoxylin and eosin (HE) staining; original magnification, $\times 1001$ and small atypical lymphocytes with perinuclear clear cytoplasm on the right (HE staining, original magnification, $\times 400$ ).
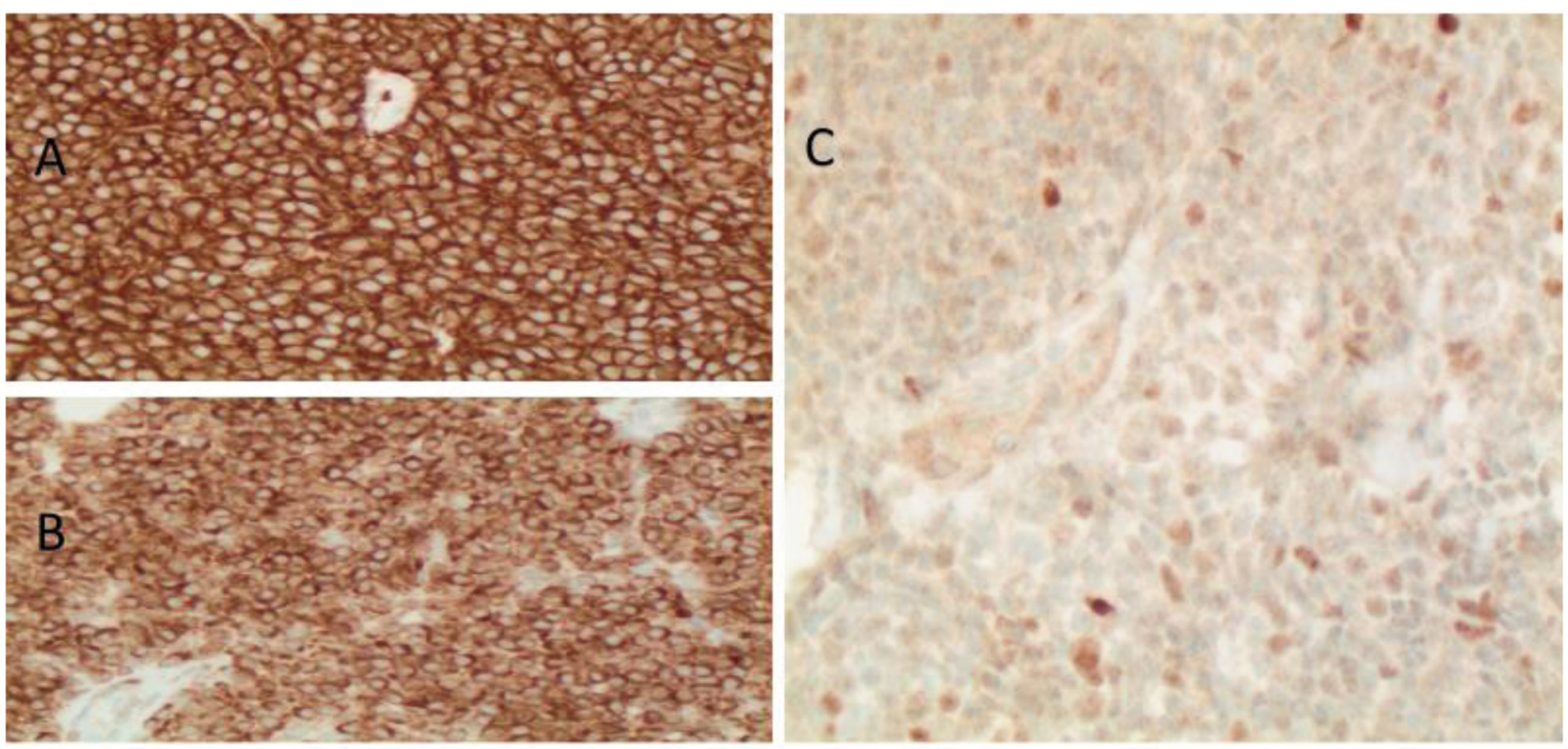

Figure 3. Immunohistochemistry slides showing lymphocytes positive for CD20 (A) and BCL2 apoptosis regulator (B), and negative for BCL6 transcription repressor $(C)$. Original magnification, $\times 400$.

et al., two out of 11 cases with advanced-stage disease were observed clinically like our case (4).

Tumor size or Ann arbor staging did not show any prognostic value for breast EMZL (4). Progression-free survival was $56-60 \%$ at 5 years and $34 \%$ at 10 years (2), (4).
Overall survival was $100 \%$ at 3 years, and $92 \%$ at 5 years (2). Disease relapse is seen in up to $37 \%$ of patients with breast EMZL within 1 year. Relapse is seen mostly at distant sites and $8 \%$ in the contralateral breast and also responds very well to treatment (2). 


\section{Conclusion}

EMZL presents rarely as primary breast lymphoma. It is a less aggressive form of breast lymphoma. Symptomatic cases respond well to radiotherapy. Asymptomatic cases can be managed with close observation as seen in our case. Overall, EMZL has a good prognosis.

\section{Conflicts of Interest}

None declared. This research did not receive any specific grant from funding agencies in the public, commercial, or not-for-profit sectors.

\section{Authors' Contributions}

The original draft of this case report was written by corresponding Author and all co-Authors helped in writing and made critical revision of the article for important intellectual content.

\section{References}

1 Talwalkar SS, Miranda RN, Valbuena JR, Routbort MJ, Martin AW and Medeiros LJ: Lymphomas involving the breast: A study of 106 cases comparing localized and disseminated neoplasms. Am J Surg Pathol 32(9): 1299-1309, 2008. PMID: 18636016. DOI: 10.1097/PAS.0b013e318165eb50

2 Martinelli G, Ryan G, Seymour JF, Nassi L, Alietti A, Calabrese L, Pruneri G, Santoro L, Kuper-Hommel M, Tsang R, Zinzani PL, Taghian A, Zucca E and Cavalli F: Primary follicular and marginal-zone lymphoma of the breast: Clinical features, prognostic factors and outcome: A study by the International Extranodal Lymphoma Study Group. Ann Oncol 20(12): 19931999, 2009. PMID: 19570964. DOI: 10.1093/annonc/mdp238
3 Thomas A, Link BK, Altekruse S, Romitti PA and Schroeder MC: Primary breast lymphoma in the United States: 1975-2013. J Natl Cancer Inst 109(6), 2017. PMID: 28376147. DOI: 10.1093/jnci/djw294

4 Ludmir EB, Milgrom SA, Pinnix CC, Gunther JR, Westin J, Fayad LE, Khoury JD, Medeiros LJ, Dabaja BS and Nastoupil LJ: Emerging treatment strategies for primary breast extranodal marginal zone lymphoma of mucosa-associated lymphoid tissue. Clin Lymphoma Myeloma Leuk 19(4): 244-250, 2019. PMID: 30686775. DOI: $10.1016 /$ j.clml.2018.12.016

5 Mendes LST and Wotherspoon A: Marginal zone lymphoma: Associated autoimmunity and auto-immune disorders. Best Prac Res Clin Haematol 30(1-2): 65-76, 2017. PMID: 28288719 DOI: $10.1016 /$ j.beha.2016.07.006

6 Shim E, Song SE, Seo BK, Kim YS and Son GS: Lymphoma affecting the breast: A pictorial review of multimodal imaging findings. J Breast Cancer 16(3): 254-265, 2013. PMID: 24155754. DOI: 10.4048/jbc.2013.16.3.254

7 Zhou C, Lv K, Lin D, Yu Y, Xu M and Bu Y: Radiological analysis of breast lymphoma: Experiences from cases series studies. Medicine (Baltimore) 98(48): e18101, 2019. PMID: 31770231. DOI: 10.1097/MD.0000000000018101

Received July 24, 2020

Revised August 14, 2020

Accepted August 26, 2020 